\title{
Beyond Proximity: Consequentialist Ethics and System Dynamics
}

\section{Erika Palmer}

Department of Comparative Politics, University of Bergen, Norway, erika.palmer@uib.no

DOI: http://dx.doi.org/10.5324/eip.v11i1.1978

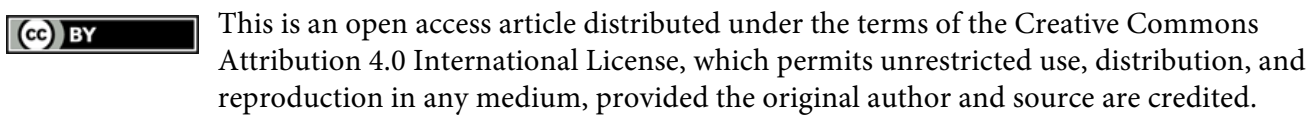

Consequentialism is a moral philosophy that maintains that the moral worth of an action is determined by the consequences it has for the welfare of a society. Consequences of model design are a part of the model lifecycle that is often neglected. This paper investigates the issue using system dynamics modeling as an example. Since a system dynamics model is a product of the modeler's design decisions, the modeler should consider the life cycle consequences of using the model. Seen from a consequentialist perspective, the consequences of policies developed from system dynamics models determine the model's moral value (ethical/unethical). This concept is explored by discussing model uncertainty from an engineering perspective. In this perspective, the ethical considerations shift from the behavior of the modeler (and away from validation) to the model itself and the model's inherent uncertainty. When the ethical considerations are taken away from the modeler and directed to what the model does, the ethical boundaries extend beyond the proximity of the model. This discussion renews the ethics conversation in system dynamics by considering this shift in philosophical perspective, and investigates how consequentialist moral philosophy applies to the modeling process and in communicating with decision-makers. A model of social assistance in Norway in the context of immigration pressures illustrates some possibilities for addressing these ethical concerns. This paper argues for an ethical framework, or at the very least, an ethical conversation within the field of system dynamics.

Keywords: System dynamics, consequentialism, philosophy of engineering, modeling ethics

\section{Introduction}

System dynamics is a field that applies systems modeling to a variety of contexts (e.g. environmental, organizational, societal) in order to develop policies to address problematic behavior in the system. System dynamics literature that explicitly addresses ethics outside of validation is scant. However, some important ethical considerations fall outside the domain of validation. The use of system dynamics modeling in public policy is an area in which the topic of ethics should be renewed. In public policy decision-making, system dynamics modeling will have an effect on 
society. The modeler must consider the effect that their decisions about subjective elements and relationships will have for people affected by the policy. The ethical concerns go beyond the normative considerations of model and policy design (the purpose of the model and policy) to the assumed causality that is necessary when making decisions in system dynamics modeling. How does one know when a model represents reality accurately enough to build policy? What happens to society if policy is built and implemented from a model that produces the correct behavior, but in the wrong way? This discussion explores these ethical concerns.

\section{What is system dynamics?}

System dynamics is a relatively new discipline, which began to develop in the mid20th century with the publication of Industrial Dynamics by Jay Forrester (Forrester, 1961). The term "system dynamics" is used when analyzing problems as a system in order to understand the feedback within the system and develop solutions. The methodology was originally developed at MIT by a group dedicated to this academic pursuit (Meadows, 2008). System dynamics is an iterative and interdisciplinary process, which views problems holistically. Essentially, using system dynamics involves identifying elements, subsystems, and the systems' context, boundaries and properties. System dynamics is both systematic and systemic in that there are systematic processes that design complex systems, but the process is rooted in systemic thinking in order to recognize and solve complex problems by seeing the whole instead of only the parts (Haskins, 2008).

System dynamics can be applied to any problem to investigate how elements operate and interact in a system. System dynamics modeling is based on ordinary differential equations. The elements in a system dynamics model consist of stocks, flows and variables (in a "stock and flow diagram"). Stocks are an accumulation of flows over time, and flows represent addition and subtraction to the stock over time. Variables in stock and flow models are elements that affect the inflows and outflows. The variables are linked to other variables and flows through instantaneous causal links. The accumulated causal behavior in the stock is affected by the flows, which are in turn affected by the variables.

The structure of a system leads to the behavior over time (accumulated in stocks), and the goal is to research all the elements and relationships in a system and put them together in order to reproduce the reference mode behavior (actual system behavior). System dynamics models include "endogenous" and "exogenous" elements. Endogenous elements are incorporated in the model structure in relation to other structural elements. Exogenous elements are variables that contain data that is directly imported into the model structure.

\section{Ethics in system dynamics}

Ethical issues in system dynamics are complicated by the philosophical foundation of the field. The field is most often understood in the context of the philosophy of science, whereby system dynamics scientifically analyzes a problem through model development. Ethical questions in system dynamics are concerned with the behavior of the modeler in the context of validation. Are the actions of the modeler ethically right or wrong when making subjective decisions about the model? Consider however that not only the behavior of the modeler is in question, but also the model itself. A developing argument in the field concerns whether system dynamics would 
be better considered as an engineering endeavor, meaning that system dynamics models are engineered artifacts. This artifact is then used to design another artifact (the policy). Using this artifact (the implemented policy) results in consequences for society (good or bad). Due to the assumed causality built into system dynamics models, not only the model (or policy purpose) but also the natural uncertainty in this methodology is ethically in question. Viewing system dynamics within the realm of engineering, the ethical discussion must consider the consequences of design because of this uncertainty. Consequentialist ethical considerations in system dynamics modeling form the point of departure for this discussion in an engineering context. However, placing system dynamics in an engineering context is debatable within the field of system dynamics. Because this placement is an important cornerstone of this ethical discussion, system dynamics as an engineering endeavor is further explored in the section "System dynamics modeling in an engineering context."

\section{Renewing the ethical discussion}

No standard procedure in system dynamics is currently in place for making ethical decisions when setting model boundaries or for making data and parameter assumptions, nor is there any stated requirement that the procedure be transparent in communicating with decision-makers. The purpose of this paper is to renew and further develop the ethics conversation within system dynamics concerning both the modeling process and in communicating with decision-makers. Developing a basic ethical foundation in system dynamics is necessary for systematically dealing with ethical considerations in modeling, which in turn further enhances the credibility of the field.

The focus of this discussion is the shift in philosophical perspective for system dynamics from science to engineering and how this affects the ethical considerations in modeling. However, there is no general understanding within system dynamics that ethics needs to be discussed or even that uncertainty in modeling exists. The first section of this paper discusses uncertainty and transparency issues in modeling as grounds for having an ethical conversation in system dynamics. Next, the discussion explores how system dynamics' philosophical foundation frames the ethical discussion, which calls for ethical questions to be thought of in a new way, through the context of consequentialism. The paper then illustrates this context with a model of social assistance in Norway related to immigration policy pressures, ending with a discussion of topics for further research.

\section{Uncertainty and transparency}

The nature of system dynamics modeling breeds uncertainty. The modeler decides whether or not this uncertainty is made transparent, which is an issue that deserves attention. Transparency depends on the ethical behavior of the modeler, though it is the model itself that is ethically charged. There are many reasons why a modeler might not want to communicate the uncertainty of their model. Reasons could include that the audience is funding the project; the modeler does not want to show weakness; or the modeler has an ulterior agenda. The issue under discussion is not how this uncertainty is evaluated (see Walker, Harremoës, Rotmans, van der Sluijs, 
van Asselt, Janssen, and Krayer von Krauss (2003) for a framework in which to address this), but why modelers must consider uncertainty beyond the validation of their models.

\section{Assumed causality and validation}

The issue of causality is blurry in system dynamics, especially when communicating with those outside of system dynamics. System dynamics models represent "operational behavior"; modelers observe relationships in a system and make assumptions about instantaneous causal relationships, which goes against scientific principles of causality. Using the word "causality" creates communication problems with decision-makers as well, because the scientific meaning is often assumed. The system dynamics causality issue is both semantic and philosophical. What system dynamicists mean by "causal" is different from the scientific definition (a natural law). System dynamics models show how variables operate in relation to flows. Models are one possible structural explanation and not a causal declaration, meaning the models show the "how" (and only one possible how) and not the "why." This does not mean that system dynamics methods should be practiced any differently than what is the current practice; it does, however, mean that we need to think differently about the methods, which is the philosophical part of the issue.

Models, as a set of aggregated causal assumptions ("observed operational relationships") - and regardless of validation - are by definition uncertain, because causality is assumed. We speak of "robustness" when evaluating the uncertainty in system dynamics models. To make a model as robust as possible, modelers validate the model. There are various levels for validating models, from the technical validation to the justification of variables. It is assumed that modelers will make sure that their models are as robust as possible (having the lowest possible level of uncertainty) through validation, and decision-makers assume that the modeler has done this. Because of this assumption, the model's robustness and related issues are not necessarily communicated to those making decisions based on the outcome of the model.

The lack of ethical guidelines for system dynamics begs the question: How can the field of system dynamics trust practitioners to do the right thing? How is the decision-maker to understand that the causality is assumed? What level of transparency do practitioners offer in reality? How can the field of system dynamics know that everyone is practicing the methodology consistently and acting as ethically as possible? As explained by Forrester (2007), many people who build models are not skilled system dynamics practitioners. Because system dynamics software is so easily learned, and many people outside the field use system dynamics modeling, would it not strengthen the field to have ethical guidelines that practitioners must follow in order to call themselves system dynamicists?

Many in the field may think that ethical considerations are not an issue and resist a conversation on the topic. "Show me a model that has had a negative effect on society" or "Professional system dynamics practitioners already follow validation procedures" are two common criticisms I have heard in response to the idea of discussing ethical issues in system dynamics. However, is validation enough to remove uncertainty? Valid models still assume causality. Does the nature of system dynamics, with its assumed causal relationships, require further ethical consideration concerning the societal impact of the model? No matter how valid or 
robust a model is, the model has aggregated causal assumptions. This does not mean that the model is wrong or not useful; it does however mean that system dynamicists must consider the model in a different light and ask different ethical questions.

Ethical concerns in modeling are also important to consider because system dynamics modeling lacks policy design and implementation focus. Explanatory models may be uncertain, but this is not the main problem for these models in terms of potential consequences for society. The frequent absence of a developed policy model structure built to change behavior presents a larger issue, as explained in the following section.

\section{Policy design and implementation}

Wheat (2010) explains that system dynamics models are often merely concerned with policy design as parameter testing and not with policy implementation. At best, adjusting parameters as policy design results in poor feasibility and possible policy implementation failure. At worst, it means negative consequences for society. However, in addition to building policy structure beyond parameter testing to avoid either of the above scenarios, understanding and communicating inherent uncertainty must be considered as well.

Uncertainty is outside the ethical realm of the policy model's purpose. "Model purpose" holds normative ethical considerations that do not include uncertainty. The focus of this discussion is the uncertainty in every system dynamics model, which has the possibility of negative consequences for society regardless of the normative implications of the policy purpose. The ethical issue at hand concerns the consequences, or the unintended effects of an implemented policy, generated by a model where the modeler establishes subjective elements and relationships as causal when in reality they are not.

System dynamics modeling produces just one possible design to explain behavior, and policy models designed from the explanatory models will only be successfully implemented if the explanatory model's assumptions are correct and if implementation issues are fully considered. Because uncertainty is inherent in the process - and does not negate the model's usefulness - this must be made transparent to those who make decisions about policy implementation.

\section{System dynamics modeling in an engineering context}

Within system dynamics, models are considered causal mathematical models that represent a theory of an actual system. Each causal claim in the model must be supported, and if critics disagree with one equation, then the entire model is disregarded (Barlas, 1990). Therefore, from the perspective of the philosophy of science, seeking justification for causal claims in a system dynamics model can be difficult. How does one ever truly know when a relationship is causal, and how far does one have to go to support the causal claim? The truth is that, as modelers, we cannot know if we have represented causal relationships in our models. We assume causality in our models, which gives rise to one of many designs of the structure that produces the behavior.

Science aims to understand ("to know") a system, whereas engineering is steeped not in the truth or falsehood of a system, but in how it operates ("the how"); the 
knowledge that versus the knowledge how (Schmidt, 2012). System dynamics models are designed to understand how a system works, not to understand that (why) it works. Olaya (2014) explains how science is very different from engineering (although engineering contributes to science, it does not use the scientific method), and that system dynamics is in fact an engineering enterprise. System dynamics was born out of engineering and applied to management science. However, system dynamics has deviated from its engineering heritage by seeking to justify itself as science by using the scientific method. If system dynamics is applied as an engineering endeavor, the model does not claim causality. The model is used to understand how the system works - as one way among many structural designs making it useful to decision-makers as a tool for gaining insight into complex systems.

Because engineering and system dynamics are both concerned with design, system dynamics should concern itself with the operations of the system and not the causal relationships. Olaya (2012) states:

Operational thinking opposes to mere theorizing activities based on dataanalysis, which happens to be the fashionable way (and the "scientific" style in many cases) to study social systems. Instead of developing knowledge by observation to generate general statements through induction, the production of knowledge through operational modeling does not rest on data in order to bring understanding or explanation. Rather, it relies on the generation of dynamic hypotheses that explain the performance of a system in function of its structure that is generated by its operations. Such an approach recognizes human systems as systems that change through time according to free actions of decision makers (2).

System dynamicists design models that represent social systems in order to develop policy to improve a system. The models are "intangible artifacts" designed by the modeler, and this artifact is then used to design other artifacts. Models are used to design policy; policy includes an entire array of artifacts, such as regulations, plans and organizational structure. Trademarks of engineering first and foremost include design and operations (knowledge how), but also the use of heuristics, making decisions, being creative, using trial and error methodology, having purpose versus being impartial and being particular rather than universal (Goldman, 2004). All of these are also trademarks of system dynamics, making it easy to see the link between system dynamics and engineering (Olaya, 2014). This paper does not attempt a fullscale examination of whether system dynamics is engineering or science. However, if we think of system dynamics as engineering, how does this impact validation and ethics?

Science is very concerned with ethics as regards the behavior of scientists and the validation of their methods. Validation is irrelevant in engineering. An engineered artifact is not true or false. It either technically works or it does not. In engineering, ethical considerations fall outside of validation and the behavior of the practitioner. The purpose of the artifact and uncertainty (robustness) in design are the ethical concerns in engineering. Why was the artifact designed, and how will this affect society (the purpose)? Because the artifact does not claim knowledge of why cause and effect occurs, what is the risk of consequences to society from the uncertainty (robustness) in design? As mentioned earlier, the normative implications of model 
purpose are not under examination in this paper, as this concerns the nature of specific case studies. In the more general examination of moral philosophy and system dynamics, given a philosophical foundation in engineering, system dynamicists must consider the societal consequences of the uncertainty in their models.

\section{Consequentialism and system dynamics modeling}

Assuming that system dynamics is an engineering enterprise, what ethical issues must be considered? And why does uncertainty take center stage?

In an engineering context, the ethical dilemmas of the modeling process concern the consequences that arise because of model design, which only surface after the recommended policies of the model have been implemented in society. System dynamics modeling of social systems is no easy task. Uncertainty exists in many variables, making this a difficult ethical undertaking.

Various moral philosophies could be applied when evaluating decision-making in modeling. This paper does not attempt to explain these different philosophies and how they can be used in system dynamics. Rather, it attempts to illustrate the societal consequences that can arise due to the nature of system dynamics modeling, i.e. a consequentialist approach. Pruyt and Kwakkel (2007) provide a good overview of different moral philosophy approaches in system dynamics.

The foundation for the ethical exploration in this discussion is consequentialism. The discussion does not intend to provide a comprehensive explanation of consequentialism or its merits and criticisms. Consequentialism serves in this discussion as a basis for understanding the ethical issues in system dynamics modeling, and to this end a brief overview of the concept of consequentialism is provided.

Consequentialism is a moral philosophy where, in its simplest and purest form, the best decision in any given situation is the one that provides the greatest benefit overall, as judged from an objective standpoint (Scheffler, 1988). In this way, the moral value of a decision is based on the net benefit it has for those affected by the decision. Many different moral theories are considered consequentialist, all of which - to a greater or lesser degree - have arisen out of "classic utilitarianism" (SinnottArmstrong, 2003). Utilitarianism holds that the moral worth of an action is determined by maximizing the good and minimizing the bad. However, what is considered good? In a hedonistic view, the moral worth of an action is determined by the amount of pleasure it produces and the amount of pain it avoids. This is rather simplistic and leads to situations that are morally irresolvable (SinnottArmstrong, 1988). In a pluralistic view of consequentialism ("pluralistic utilitarianism,") many goals can be used to assess the moral value of a decision, which helps to alleviate morally irresolvable dilemmas in "hedonistic utilitarianism."

There is also the issue of what is considered a "consequence." Classic utilitarianism requires all consequences of a decision to be known before a decision is made. This is an impossible task, and various theories of consequentialism have fashioned ways to reconcile this problem. Of importance to this discussion of uncertainty and modeling, however, is the distinction between "unwanted" versus "unintended" consequences (Koehn, 2010). Unwanted consequences are foreseen consequences, and the decision-maker chooses to avoid them (morally good) or not 
(morally bad). Unintended consequences, however, are unforeseen consequences that the decision-maker cannot control. In the case of system dynamics modeling, unintended consequences are the result of uncertainty in the model.

Models cannot be $100 \%$ robust, with all uncertainty removed, because the nature of system dynamics requires assumed causality. Therefore all models have the potential for harm, i.e. negative unintended consequences due to uncertainty. This is why communicating uncertainty to decision-makers is essential. Practitioners can only give the best of what system dynamics can offer - unbiased, well-researched models; decision-makers have the moral responsibility for policy implementation, and they must understand the risk of implementing policy built and tested on models that have inherent uncertainty.

Validation of causal claims is a matter of professional ethics found in the sciences, which concerns the behavior of the scientist. The outcome of the causal assumptions in system design and what this means for citizens and society are a matter of consequentialist ethics in engineering. In an engineering perspective, system dynamics models do not need to be validated because they do not attempt to explain causality. At the same time, system dynamics models must be designed with the least amount of uncertainty to avoid negative downstream societal consequences. This is where the ethical distinction is made between the modeler and the model. In engineering, the modeler's behavior is no longer called into question concerning methods, but instead the model itself is called into question for the potential impact it could have on society.

Determining where the ethical responsibility lies is less of a problem in engineering disciplines compared with the field of system dynamics. In engineering, the relationship between engineering models and moral philosophy is represented at least to some extent in the literature (e.g. Herkert, 2000; Jenkins, 2015; Katz, 2011). But since system dynamics is considered a field of scientific study, the exploration of ethics in an engineering context is lacking. It may not seem immediately apparent why this ethical discussion should focus on uncertainty in models and its consequences for society. Uncertainty in modeling is nothing new, and a system dynamics model can suffer from a host of ethical concerns in addition to uncertainty. These issues include: individual bias (which can never be fully avoided), the issue of sponsorship of the modeling project and a lack of transparency with decision-makers. However, these are ethical concerns related to the behavior of the scientist (modeler), not the artifact (model).

As mentioned, system dynamics has a dearth of ethical literature, and ethical concerns related to the behavior of the scientist should indeed be explored. However, system dynamicists must also go beyond their own ethical behavior and consider the model an "ethically charged artifact" because of the potential harm it may have for society, which is why consequentialism is relevant in this discussion. Even though a modeler may be acting as ethically as is humanly possible (in an unbiased and completely transparent fashion in an objective environment), once the model leaves their hands and is used by decision-makers, the model is a tool that can produce harm. It should also be noted that it is very easy for system dynamicists to fall into the trap of believing their model is $100 \%$ robust with no uncertainty once the model is validated. Uncertainty is always present, and the model may have unintended consequences for society once it becomes independent of the modeler. 
Shifting the focus away from the modeler and onto the model requires the modeler to expand their ethical awareness beyond the modeling process. In engineering, this is termed the "product life cycle." So too should system dynamicists consider their model's life cycle.

\section{Beyond proximity}

As the model is a product of the modeler's decision-making in design, the modeler should consider the "life cycle consequences" of using the model. In this regard, the consequences of the policy (given the level of uncertainty on which the model is built) determine the moral value (ethical/unethical) of the model from a consequentialist perspective. Philosophy of engineering ethical considerations relate to the constructed artifact and the effect this has on society. When engineering social systems, the model and the policy are the artifacts. The consequences of the model design serve as the modeler's ethical guide. What are the causal assumptions? What other design options are possible? What are the risks if the assumptions are wrong, i.e. will the model be used to develop policy? Will the policy developed from the model create harm for society if the assumptions are indeed incorrect? It is not always possible to know these answers, but the modeler should ask them and believe that the model is as unbiased as possible with the greatest amount of input from all relevant sources. Professional system dynamics practitioners may understand this as a given. However, as mentioned above, not all people using system dynamics are professional practitioners.

When the ethical considerations are removed from the model itself and placed on what the model does, the "ethical boundaries" are extended beyond the proximity of the model.

The concept of ethical boundaries is very well expressed by Bowen (2009):

It may be proposed that the articulation of an aspirational engineering ethic can be facilitated by extending the I-You vocabulary beyond proximity, to include a relationship with people who may be distant in place and/or distant in time. Thus, the task of the engineer may be viewed as the development of technical knowledge and technical activities, the world of I-It, in response to an I-You concern for those benefiting from the technical advance. The people affected by the activities may be located far from the place where the engineering work is conceived and planned. In some cases, they may be far from the place where the engineering artefacts are constructed or even far from the place where the completed, engineered artefacts are located (140).

In avoiding negative downstream consequences, thereby attempting to gain moral value, it all comes back to the issue of communication. Decision-makers claim the ethical role in policy implementation. They must know what the causal assumptions are in both the explanatory and policy models. When design justification is difficult, the modeler may be less inclined to make the assumptions known to the client or peers. However, making these justification break-offs explicit in communicating the model and as part of the ethical considerations in design is essential. In the words of Ulrich (1987): "As long as he does not learn to make transparent to himself and to others the justification break-offs flowing into his designs, the applied scientist cannot claim to deal critically with the normative content of these designs" (277). 


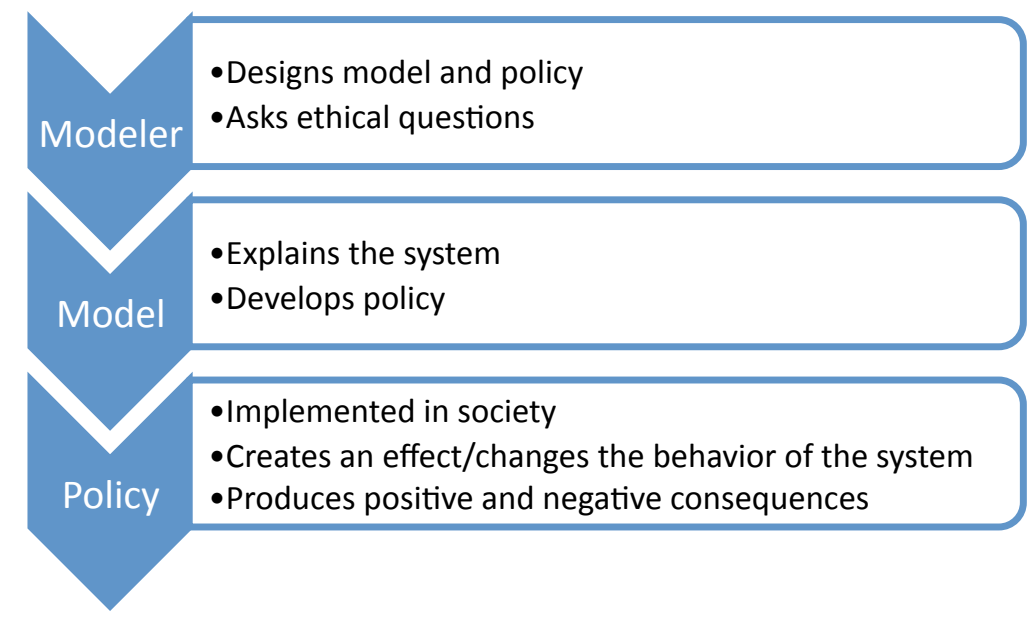

Figure 1: The modeler's behavior leading to artifacts and the effect of these artifacts on society.

Figure 1 illustrates the expansion of the "ethical horizon" and the scope of influence of the modeler. The artifacts (model and policy) themselves are neutral in the philosophy of science. Scientific artifacts (theories) are either right or wrong, not ethically good or bad. Ethics in this sense comes back to the behavior of the scientist, not the theory itself. This is the opposite in engineering, where the artifacts themselves are ethically charged.

\section{Asking the ethical questions: System dynamics and the Norwegian welfare state}

Expanding the boundaries of what comes under ethical consideration is easy in a philosophical discussion, but what would this look like in practice? The following list is an example of possible ethical questions that consider the consequences of model design.

\begin{tabular}{|c|c|}
\hline $\begin{array}{l}\text { In designing and } \\
\text { building the }\end{array}$ & $\begin{array}{l}\text { How well supported are the causal assumptions? What other structural options could } \\
\text { produce the behavior? }\end{array}$ \\
\hline & Have I made all possible input to the model as objective as possible? \\
\hline
\end{tabular}

Have I introduced bias into the model? How accurate a representation of society is the model? What is the level of uncertainty (robustness)?

\begin{tabular}{|c|c|}
\hline \multirow{4}{*}{$\begin{array}{l}\text { Pushing beyond } \\
\text { proximity } \\
\text { (beyond the } \\
\text { initial artifacts) } \\
\text { requires } \\
\text { modelers to ask } \\
\text { questions such }\end{array}$} & $\begin{array}{l}\text { What will the policy do to society if the causal assumptions in the structure are wrong? } \\
\text { What is the level of risk that the model is inaccurate? }\end{array}$ \\
\hline & Have I communicated the uncertainty to decision-makers? \\
\hline & $\begin{array}{l}\text { Does the policy produce the good for which it was intended? Are there unintended } \\
\text { negative side effects? }\end{array}$ \\
\hline & Do the side effects of implemented policy indicate that the model design is inaccurate? \\
\hline
\end{tabular}


Armed with these questions, let us take a closer look at how this would be applied in a project about the Norwegian welfare state.

\section{Norwegian social assistance and immigration}

A model of Norwegian social assistance in the context of immigration patterns illustrates how these questions can be applied. As in all modeling projects, this study requires making decisions about subjective elements and relationships in the system and subsystems.

Consider the following simple model of the number of people on social assistance (unemployment support) in Norway with a focus on immigration (Figure 2).

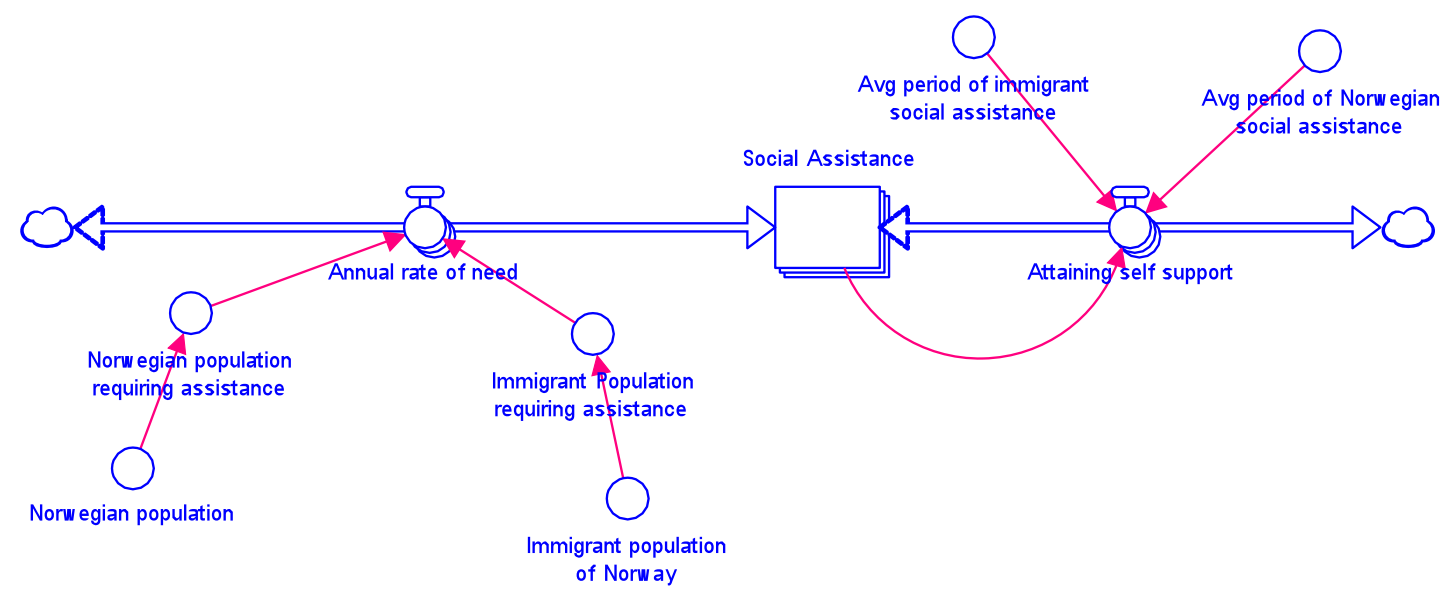

Figure 2: Simple stock and flow representation of the cost of social assistance in Norway, split between Norwegian and immigrant populations.

Building a simple model, and aggregating many variables, makes subjective decisions not just implicit, but invisible. For example, decision-making concerning the variable "Immigrant population" makes the assumption that all immigrants are homogeneous, although they can be refugees, labor migrants, students or migrating for family reunification purposes.

In the model (Figure 3) below, the immigrant population is disaggregated. Student immigrants are taken out because they are usually not eligible for social assistance in Norway (they must prove they have sufficient funding before they are granted a visa.) Refugee regulations explain that refugees are completely supported by social assistance when they arrive, and there is no maximum time for receiving support; therefore they are assumed to have a high dependence on social assistance. It is assumed that those with family reunification visas have a low social assistance dependence, because in order to get a visa, they must show they have economic support from the family member in Norway. Labor immigrants are also assumed to have low social assistance dependence because the labor immigrants have work contracts when they arrive in Norway. All of these assumptions are justified by Norwegian immigration regulations.

For the purposes of this discussion, let us assume that all other variables besides labor, family and refugees accurately reflect reality, and that the model behavior replicates the system behavior, even with the assumptions made for labor, family 
and refugees. Let us also assume that this model resonates with the research team, and technical validation protocol has been fulfilled.

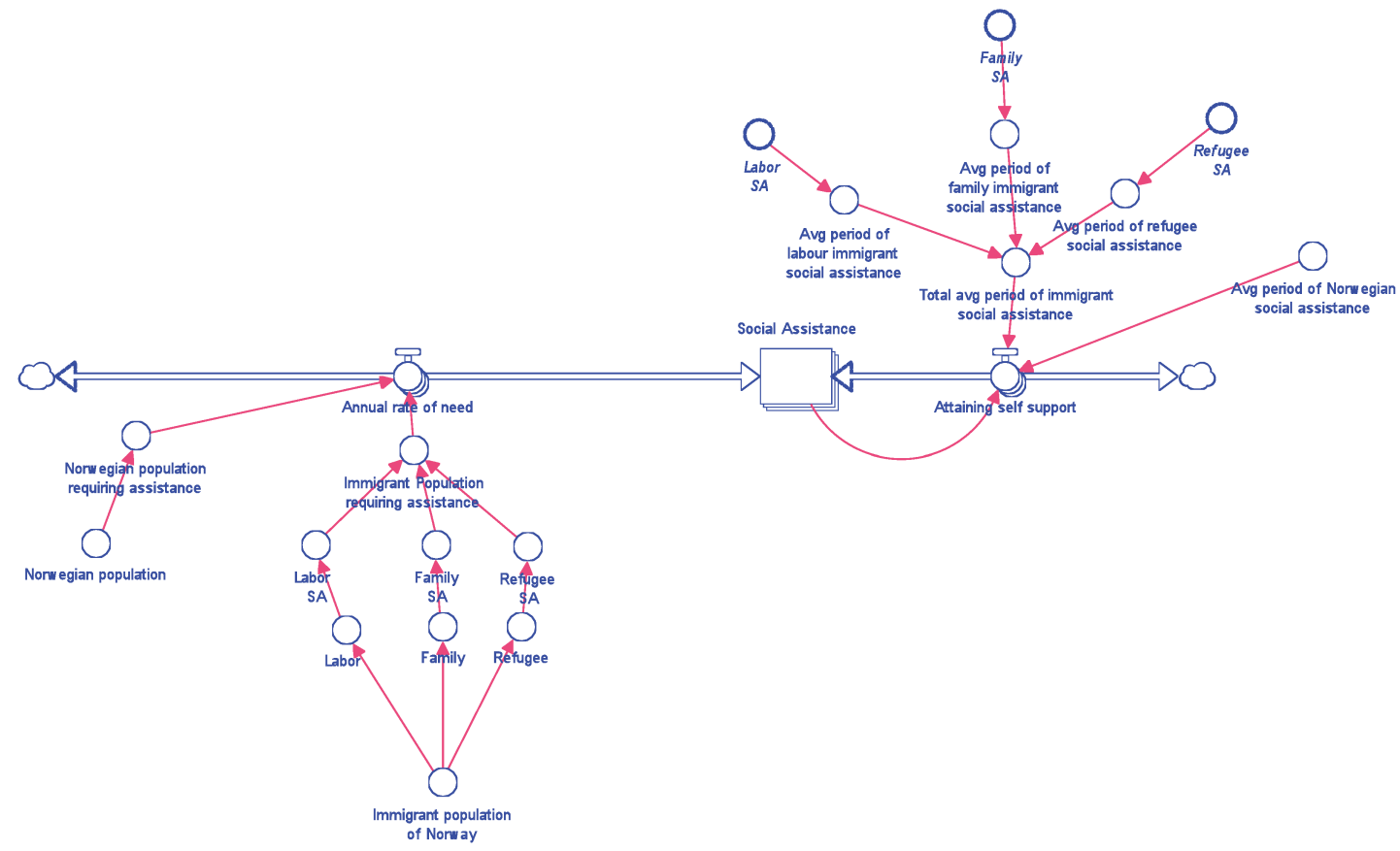

Figure 3: Disaggregation of the variable "Immigrant population requiring assistance"

The desired state of the system is fewer people on social assistance, so a policy is introduced to the model that speeds up the process of getting refugees off social assistance through an enhanced assimilation program. Since assimilation support is already available for refugees in Norway, this policy strengthens the process. Figure 4 shows this policy structure in red as the "Enhanced Assimilation Program" variable, which represents a larger policy model structure.

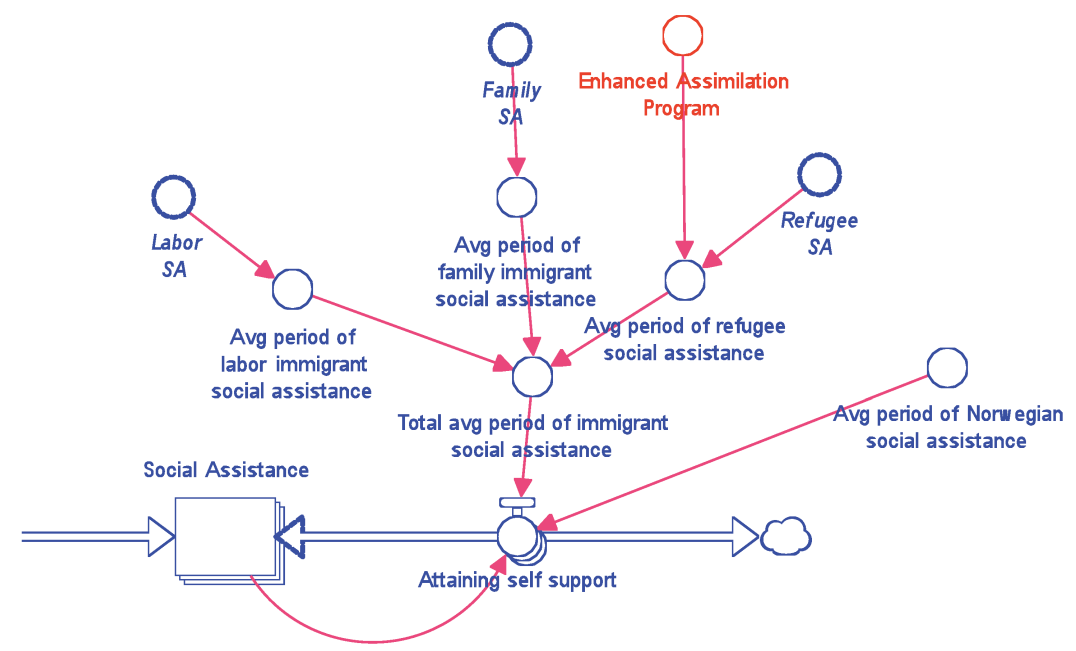

Figure 4: Cut-out of model showing the "enhanced assimilation program" policy

Let us assume that this policy has then been implemented in the Norwegian welfare system. However, the total number of people on social assistance has increased after 
implementation. The length of time that refugees are on social assistance has appeared to stay the same as indicated by unchanged unemployment rates among refugees, but for some reason the number of people needing social assistance has increased (model controlled for population increase).

In this example, the artifact (the model) is designed by the modeler, which produces an artifact (the enhanced assimilation program) that may have produced negative consequences for society. A couple options are available as a next step. The modeler could return to the model and make more assumptions about the system in order to develop a new policy. This would change the original artifact. The modeler could change the policy structure to produce the desired effect, changing the product of the original artifact. However, using an ethical framework in the first design process could have improved the probability of avoiding the negative effect (see Figure 5). Using the questions mentioned at the beginning of this section would be a place to start.

- How well supported are the causal assumptions? What other structural options could produce the behavior?

Labor and family populations were assumed to have low rates of social assistance, and refugees were assumed as having a high rate of social assistance. This is supported by immigration regulation requirements for visa applications. What other academic disciplines would have data concerning this? What sociological research is available on rates of social assistance usage by immigrant type?

- Have I made all possible inputs to the model as objective as possible?

Have I reached out to the refugee/labor/family immigrant populations to understand what their needs are? Could group model building help in understanding personal thresholds for seeking social assistance?

- Have I introduced bias into the model? How accurate a representation of society is the model? What is the level of uncertainty (robustness)?

Are cultural factors missing because the modeler is from a different culture than the immigrant populations? For example, is a social stigma associated with seeking social assistance found in some cultures and not in others? Have all alternative explanations been researched? Is labor migrant social assistance perhaps higher because workers are coming on short-term work contracts? Perhaps it is better financially to receive social assistance in Norway than to earn a normal wage in their home country. Could refugee social assistance drop substantially after an initially high period of public support?

- What will the policy do to society if the causal assumptions in the structure are wrong? What is the level of risk that the model is inaccurate?

What is the possible risk of harm to the system by implementing the policy because of uncertainty in the model representing society? If the causal assumptions are incorrect, in addition to the cost to the state, how will refugees and social support agencies suffer through an enhanced assimilation program?

- Have I communicated the uncertainty to decision-makers?

Do social support administration and funding agencies and refugee support agencies (i.e. the decision-makers) know that this model is only one of many structural designs that could lead to the system behavior? Even if the policy option of an enhanced assimilation model was the decision-maker's idea, they 
must be made to understand that it was tested on a structure built with assumed causality (i.e. a level of uncertainty, not completely robust).

- Does the policy produce the good for which it was intended? Are there unintended negative side effects?

In this example, the answer to the first question is no. The policy did not reduce the number of people on social assistance. The number of people on social assistance is rising; so the answer to the second question is yes, there are possible unintended side effects. The rising number of people on social assistance could, however, be originating from another reason altogether.

- Do the side effects of implemented policy indicate that the model design is inaccurate?

This should always be explored as a possibility. There is no definitive way of knowing whether the model is reflective of reality.

The answers to these questions and the ethical concerns raised seem obvious because this is a simple model built for explanatory purposes. Imagine, however, a large complex model or practitioners that have only just begun using system dynamics methods, or students learning how to build system dynamics models. Would it not help to have an ethical foundation in system dynamics in such cases?

Considering ethical issues must become part of the modeling process. These procedural (modeling) questions lead to the ethical questions. Figure 5 illustrates the relationships between asking ethical questions and the modeling process.

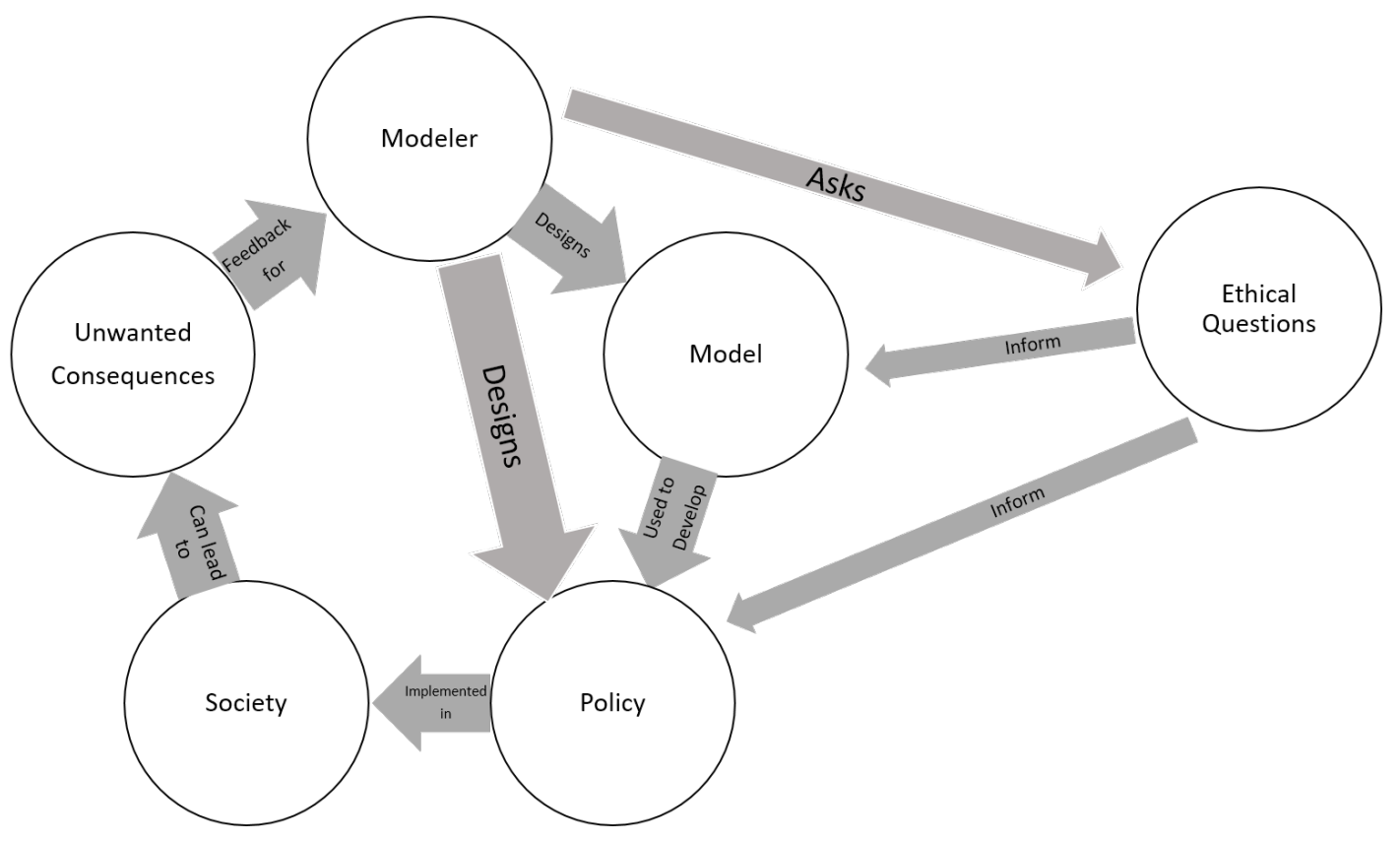

Figure 5: Relationships between the modeling process and ethical questions

\section{Stepping Forward -The Ethical Conversation in System Dynamics}

This discussion argues for an ethical framework, or at the very least, an ethical conversation within the field of system dynamics. The road to an ethical framework in system dynamics must be developed over time through discussions in moral theory and practical application. The hope is that over time a framework will 
develop into established norms, where each practitioner knows what is ethically expected of them, and those new to system dynamics are expected to uphold a certain ethical standard. Because the approaches to system dynamics methodology are varied, and many people are practicing system dynamics who are not in the system dynamics community, ethical standards of practice offer a strategy for cohesion among these diverse practitioners.

\section{Further Research}

This discussion has centered on uncertainty and how to handle it from an ethical point of view. However, it should be noted that, while many system dynamics practitioners validate their models to reduce uncertainty, there are methods in system dynamics that embrace uncertainty and use it as grounds for exploration (e.g. Exploratory System Dynamics Modelling and Analysis (ESDMA)). Further system dynamics research in ethics could investigate different system dynamics methods and the implications they have for society. "Group model building," in which users of the model form an active part of building the model, is another system dynamics methodology that is worth investigating from an ethical perspective. It can be argued that those who build and then use their own models are "making their bed and lying in it," meaning that the consequences from the policy implementation resulting from the model design would affect the designers. This opens the door for an ethical loophole to arise, whereby the system dynamicists could assign the ethical responsibility to the group instead of to themselves or to the model.

\section{Conclusion}

Engineering philosophy provides a unique perspective on ethics in system dynamics. Uncertainty in design has been addressed in system dynamics as something that must be reduced through validation. However, since structural design is variable (even if valid), modelers must ask ethical questions regarding the consequences of design uncertainty; and at a minimum, modelers must make the uncertainty transparent. The extension of ethical boundaries from the modeler to the model leads to tighter control over the societal impact of system dynamics models. The question of whether a model is scientifically valid then shifts to a question of whether the model has the potential for harm. Considering the arguments explored in this discussion, it is imperative that the field of system dynamics continues the discussion of ethics for the sake of the field's own credibility across disciplines.

\section{References}

Barlas, Y., Carpenter, S. (1990). Philosophical roots of model validation: two paradigms. System Dynamics Review, 6(2), 148-166. https://doi.org/10.1002/ sdr.4260060203

Bowen, W. R. (2009). Prioritising people: outline of an aspirational engineering ethic. In Poel, I., Goldberg, D. (Eds.) Philosophy and Engineering: An Emerging Agenda (pp. 135-146). Springer. https://doi.org/10.1007/978-90481-2804-4_12

Forrester, J. (1961). Industrial Dynamics. Cambridge, MA, MIT Press. 
Forrester, J. W. (2007). System dynamics-the next fifty years. System Dynamics Review, 23(2-3), 359-370. https://doi.org/10.1002/sdr.381

Goldman, S. L. (2004). Why we need a philosophy of engineering: a work in progress. Interdisciplinary Science Reviews, 29(2), 163-176. https://doi.org/ 10.1179/030801804225012572

Haskins, C. (2008). Systems engineering analyzed, synthesized, and applied to sustainable industrial park development. (Doctoral Dissertation, Norwegian University of Science and Technology). Trondheim, Norway: Norwegian University of Science and Technology.

Herkert, J. R. (2000). Engineering ethics education in the USA: Content, pedagogy and curriculum. European Journal of Engineering Education, 25(4), 303-313. https://doi.org/10.1080/03043790050200340

Jenkins, S. (2015). Engineering Ethics Survey: What's Your Opinion? Chemical Engineering, 122(10), 50-55.

Katz, E. (2011). The Nazi Engineers: Reflections on Technological Ethics in Hell. Science and Engineering Ethics, 17, 571-582. https://doi.org/10.1007/s11948010-9229-z

Koehn, D. (2010). Living with the dragon: acting ethically in a world of unintended consequences. New York: Routledge.

Meadows, D. (2008). Thinking in Systems: A Primer. Chelsea Green: White River.

Olaya C. (2012). Models that include cows: the significance of operational thinking. Proceedings of the 30th international conference of the system dynamics society. University of St. Gallen, St. Gallen, Switzerland.

Olaya, C. (2014). The Scientist Personality of System Dynamics. Proceedings of the 32nd International Conference of the System Dynamics Society, Delft University of Technology, Delft, The Netherlands.

Pruyt E., Kwakkel J. (2007). Combining System Dynamics and Ethics: Towards More Science? Proceedings of the 25th International Conference of the System Dynamics Society, Massachusetts Institute of Technology, Sloan School of Management, Cambridge, Massachusetts USA.

Scheffler, S. (1988). Consequentialism and its Critics. Oxford, Oxford University Press.

Schmidt, J. A. (2012). What Makes Engineering, Engineering? In Carrato, J., Burns, J. (Eds.), Structures Congress Proceedings (pp. 1160-1168). American Society of Civil Engineers. https://doi.org/10.1061/9780784412367.104

Sinnott-Armstrong, W. (1988). Moral Dilemmas. Oxford, Blackwell.

Sinnott-Armstrong, W. (2003). Consequentialism. In: The Stanford Encyclopedia of Philosophy. Retrieved 01/08/2016 from http://plato.stanford.edu/entries/ consequentialism

Ulrich, W. (1987). Critical heuristics of social systems design. European Journal of Operational Research, 31(3), 276-283. https://doi.org/10.1016/0377-2217(87) 90036-1

Walker, W. E., Harremoës, P., Rotmans, J., van der Sluijs, J. P., van Asselt, M. B., Janssen, P., Krayer von Krauss, M. P. (2003). Defining uncertainty: a conceptual basis for uncertainty management in model-based decision support. Integrated assessment, 4(1), 5-17. https://doi.org/10.1076/ iaij.4.1.5. 16466 
Wheat, I. D. (2010). What can system dynamics learn from the public policy implementation literature? Systems Research and Behavioral Science, 27(4), 425-442. https://doi.org/10.1002/sres.1039 